\title{
Drug utilization study and prescribing patterns in psychiatry patients at a tertiary care hospital
}

\author{
Venkatesh Perumal M. ${ }^{1}$, Surendra Kumar Bouddh ${ }^{1 *}$, Nirmal S. R. ${ }^{2}$, Ashok Deshpande ${ }^{2}$, \\ Jai Singh ${ }^{2}$, Natesh Prabhu M. ${ }^{1}$
}

\begin{abstract}
${ }^{1}$ Department of Pharmacology,
${ }^{2}$ Department of Psychiatry,

Dhanalakshmi Srinivasan

Medical College (DSMCH),

Siruvachur, Perambalur, Tamil

Nadu, India
\end{abstract}

Received: 08 February 2018

Accepted: 07 March 2018

\section{*Correspondence to:}

Dr. Surendra Kumar Bouddh, Email: sk85bouddh@gmail.com

Copyright: (C) the author(s), publisher and licensee Medip Academy. This is an openaccess article distributed under the terms of the Creative Commons Attribution NonCommercial License, which permits unrestricted noncommercial use, distribution, and reproduction in any medium, provided the original work is properly cited.

\begin{abstract}
Background: The Drug utilization research (DUR) compares drug use between different countries and regions and is used to assess the rationality of prescribing pattern of the drug therapy. With this background we decided to evaluate antipsychotic drugs prescribing pattern in the psychiatric patients in a tertiary care hospital.

Methods: The study was carried out at Department of Psychiatry, DSMCH. It was open label, cross - sectional, prescribed Documents based study. Duration of the study was one month (May-2017). Out-Patient number, age, sex, diagnosis, prescribed generic name, brand name, dose, route of administration, duration of therapy obtained from the Prescription register of Out - Patient Department of the Psychiatry.

Results: The clinical experiences of the Psychiatrist I, II and III were 17 years, 35 years and 10 years respectively. The Psychiatrist I, II and III prescribed treatment for $36(31.9 \%), 61(54 \%)$ and $16(14.2 \%)$ patients respectively. Among overall $(\mathrm{n}=113)$ patients (average age 38.9 years), male $\mathrm{n}=56(49.6 \%)$ and female $=57(50.4 \%)$ were treated by all the three psychiatrists. The percentage of prescription of various drugs used were: Escitalopram (15.7\%), Clonazepam (14.6\%), Sertraline (8.7\%), Risperidone (7.5\%), Propranolol (6.7\%), Olanzapine $(6.3 \%)$, Quetiapine (5.9\%), Trihexyphenidyl (5.5\%), Amitriptyline $(5.1 \%)$ and Other prescribed drugs, were between ( 0.4 to $2.8 \%$ ).

Conclusions: From this study, it can conclude that rational usage of drugs were followed in this study. All three prescribers (Psychiatrist I, II, and III) prescriptions were found to be rationale.
\end{abstract}

Keywords: Antipsychotic drugs, Drug utilisation research (DUR), Mentally - ill, Prescribing pattern, Psychiatry, Rational use of drugs

\section{INTRODUCTION}

The Drug utilization research pioneered work done by Arthur Engel, Sweden and Pieter Siderius, Holland has compared drug use between different countries and regions. The purpose was to find the rationality of drug therapy prescribed. The Drug utilization research (DUR) was defined by the WHO in 1977 as the marketing, distribution, prescription and use of drugs in a society, with special emphasis in resulting medical, social and economic consequences. ${ }^{1}$ Periodic evaluation of drug utilization pattern need to be done to enable suitable modifications in prescription of drugs to increase the therapeutic benefit and decrease the adverse effects. Irrational drug use is a widespread problem in many countries of the world.

In $1985 \mathrm{WHO}$, for the first time defined the rational use of medicines as "patients receive medications appropriate to their clinical needs, in doses that meet their own requirements, for an adequate period of time and at lowest cost to them and their community. The rational use of drugs is based on the RULE OF RIGHT that means, the right drug, To the right patient, In right dosage, At a right cost, with right documentation. 
For a developing country like India, a National drug policy is needed to rationalize drug use. To achieve this, it is very important to determine drug use patterns and monitor drug-use profiles over time.

Psychiatry is a branch of medicine that deals with mental, emotional or behavioural disorders. Utilization pattern of drugs varies from place to place influenced by many factors.

According to National mental health survey 2015- 2016, the overall weighted prevalence for any mental morbidity was $13.7 \%$ life time and $10.6 \%$ current mental morbidity in India. These patients are a burden to the society and their family in terms of non-productive life. The expanding and challenging field of psychopharmacology is constantly seeking new and improved psychotropic drugs to treat psychiatric disorders. $^{2}$

Antipsychotics are a class of agents which are able to reduce psychotic symptoms in a wide range of conditions like Schizophrenia, Bipolar disorder, Psychotic depression, Senile psychosis, various Organic psychosis and Drug induced psychosis. ${ }^{3}$ Even drugs like antidepressants, benzodiazepines and mood stabilizers are used for symptomatic management in psychiatric disorders. During the past two decades, the development of newer drugs like Selective Serotonin Reuptake Inhibitors (SSRIs) and atypical anti-psychotics have drastically changed the drug therapy protocols. Hence, the present study was designed to outline and assess the utilization of antipsychotics and its prescribing pattern in a tertiary care teaching hospital in India.

\section{METHODS}

After obtaining the ethical approval from the Institutional Ethics Committee, the study was initiated. Study was carried out at Department of Psychiatry, Dhanalakshmi Srinivasan Medical College, Perambalur. It was open label, cross - sectional, prescribed documents based study. Duration of the study was one month (May-2017).

\section{Inclusion criteria}

All Old and New patients from the psychiatry outpatient department of all Ages and both sexes where included for the study.

\section{Exclusion criteria}

Data from the outpatients' register which were found to have Convulsions, Critical illness, Unconsciousness and those with Incomplete data were excluded.

\section{Data collection}

Out-Patient number, age, sex, diagnosis, prescribed generic name, brand name, dose, route of administration, duration of therapy, from the Prescription register of Out Patient Department of the Psychiatry were collected.

\section{Statistical methods}

The data was analyzed by using Microsoft excel and Statistical Package for Social Sciences Version: 16 software to compare the frequencies of drug utilization in reference to the diagnosis.

\section{RESULTS}

The clinical experiences of Psychiatrist I, II and III was 17 years, 35 years and 10 years respectively. The PsychiatristI, II and III prescribed treatment for 36 (31.9\%), 61 (54\%) and $16(14.2 \%)$ patients. Among overall $(\mathrm{n}=113)$ patients (average age 38.9 years), male $n=56$ (49.6\%) and female $=57(50.4 \%)$ (Figure 1$)$ were treated.

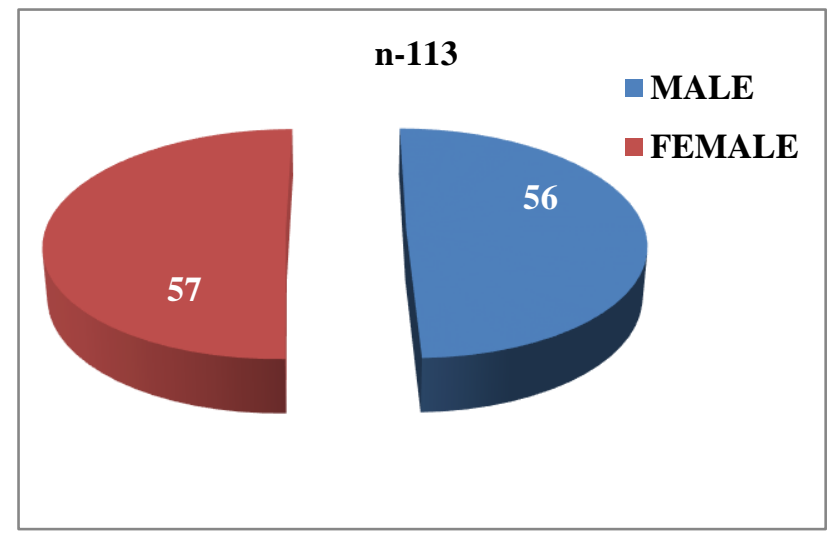

Figure 1: Demographic presentation for male/ female ratio.

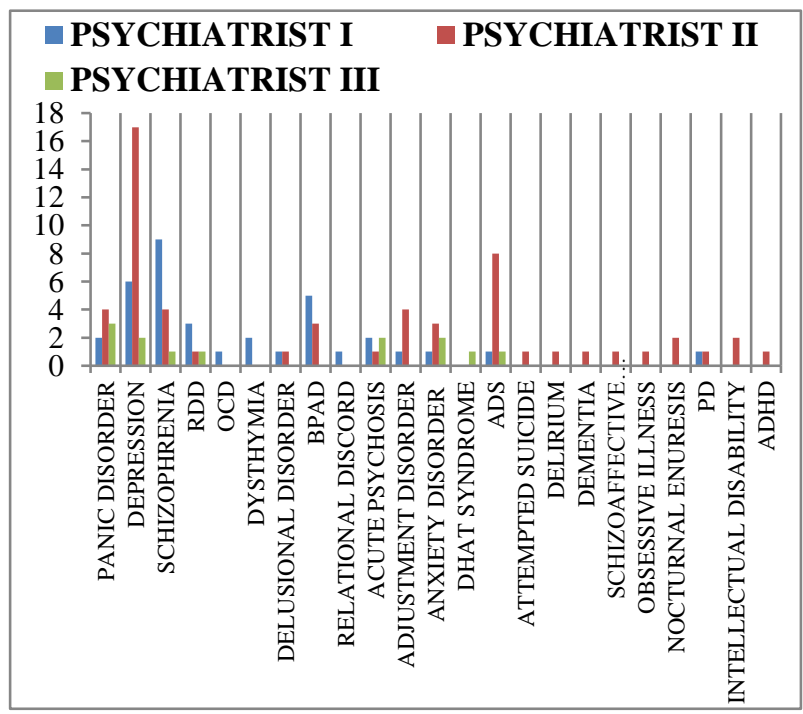

Figure 2: Demographic presentation for percentage of diseases.

The percentage of prescription of various drugs were found are Escitalopram (15.7\%), Clonazepam (14.6\%), 
Sertraline (8.7\%), Risperidone (7.5\%), Propranolol (6.7\%), Olanzapine (6.3\%), Quetiapine $(5.9 \%)$, Trihexyphenidyl (5.5\%), Amitriptyline (5.1\%) and remaining other prescribed drugs, like Carbamazepine, Chlordiazepoxide, Sodium valproate, Fluoxetine, Dothiepine, Atenolol, Disulfiram, Levetiracetam, Clozapine, Inj. Fluphenazine, Livogen, Lithium, Imipramine, Lorazepam, Baclofen, Inj Thiamine, Donepezil and Alprazolam percentage were between $(0.4$ to $2.8 \%$ ).

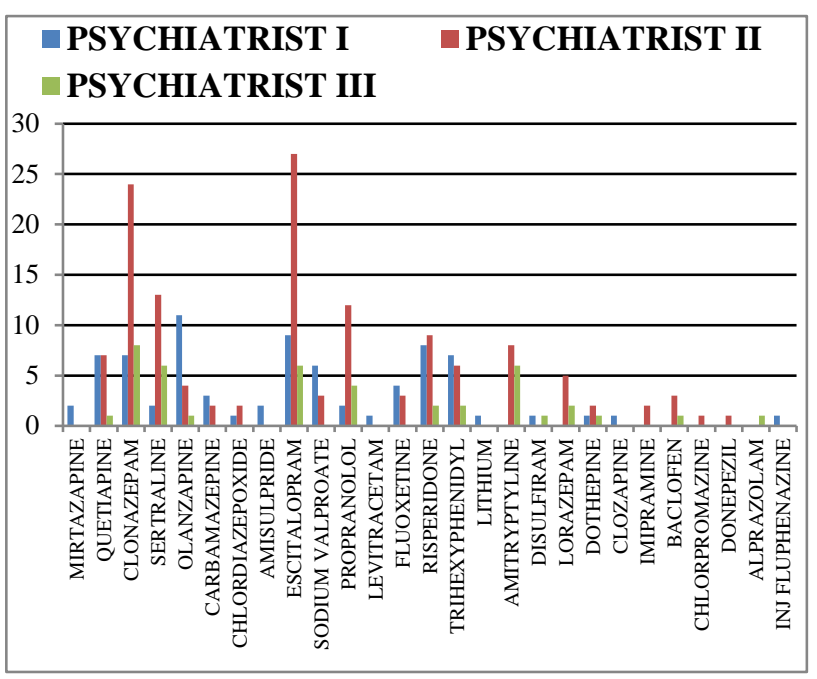

Figure 3: Frequency of drug administration.

\section{DISCUSSION}

This study was carried in the way to assess the morbidity pattern of diseases in psychiatry and the prescribing way of psychotropic drugs. More female patients visited the psychiatry OPD than male in this study. The average number of psychotropic drugs per prescription was 2.0, which was lower than that found in similar studies, where it ranged from 2.3 to 3 drugs per prescription. ${ }^{4}$ The most common diagnosis being Depression and the drug prescribed were Escitalopram. Currently, Selective serotonin reuptake inhibitors (SSRIs) are greatly preferred over the other classes of antidepressants. The adverseeffect profile of SSRIs is less prominent than that of some other agents, which promotes better compliance because current treatment guidelines recommend use of SSRIs as the first-line agents in patients with Depression. SSRIs are generally free of sedative effects and safer at high doses. Better tolerability, combined with their mild adverse effects, accounts for their popularity as most widely prescribed. ${ }^{5}$ Authors had also seen that mostly benzodiazepines namely Clonazepam were co-prescribed with all these antidepressants. In this study clonazepam was highest prescribed Benzodiazepine drug.

Among the antipsychotics Risperidone was the most common drug used along with anticholinergics in this study. Atypical antipsychotics are now rated as first-line agents because of their low propensity to cause
Extrapyramidal side effects, Efficacy against refractory cases, better tolerance, low relapse rate, and safer adverse effect profile. ${ }^{6}$ Olanzapine and Risperidone were the most commonly prescribed atypical antipsychotics, which is consistent with previous studies. Almost all prescribed antipsychotic drugs were second generation antipsychotics, Risperidone followed by Olanzapine and Quetiapine. The same pattern of prescribing atypical antipsychotics is followed in India with very less clozapine prescriptions. ${ }^{7}$ Although evidence base is very small, Olanzapine, had been showing more adverse effect than Risperidone in some studies from India., ${ }^{8,9}$ Selecting Risperidone as a first choice is right decision. In another part of India, typical antipsychotic drugs like Haloperidol, Amisulpride, Trifluperazine, Blonanserine, Thioridazine, Loxapine, Chlorpromazine were also being prescribed along with Atypical antipsychotic drugs as a first choice. ${ }^{10}$

Out of total Anti-anxiety drugs prescribed, majority were Benzodiazepines, which is consistent with that reported in previous studies. Benzodiazepines can be reasonably used as an adjunct in the initial stage, while SSRIs are titrated to an effective dose and they can be tapered over 4-12 weeks were the SSRI is continued. Alprazolam was not prescribed to any patient suffering from anxiety disorder or otherwise, and rightly so because of its higher dependence potential. Alprazolam has a short half-life, which makes it particularly prone to rebound anxiety and psychological dependence. In the present study, Clonazepam were the highest prescribed Benzodiazepine drug and has become a favoured replacement because it has a longer half-life and empirically elicits fewer withdrawal reactions upon discontinuation. From this data it is evident that whatever data signifies the prescription pattern can also depend on the availability of drugs in that particular hospital setting or dispensary and also will be dependent on the locality which may also be one of the factors that will influence the prescribing patterns. ${ }^{10}$

\section{CONCLUSION}

The prescribing pattern from all the psychiatrists were found to be rationale. Depression was the commonest diagnosis encountered followed by schizophrenia. Escitalopram was the most common medication used for depression and Risperidone was the most common medication used for Schizophrenia followed by Olanzapine.

\section{ACKNOWLEDGEMENTS}

Authors would like to thank all the healthcare professionals of Departments of Pharmacology and Psychiatry, Dhanalakshmi Srinivasan Medical College, Perambalur, India and the members of Institutional Ethics Committee (IEC) of Dhanalakshmi Srinivasan Medical College India for their constant support and guidance.

Funding: No funding sources

Conflict of interest: None declared 
Ethical approval: The study was approved by the Institutional Ethics Committee

\section{REFERENCES}

1. WHO. Introduction to Drug Utilization Research. 2003:33.

2. Kaul VR, Beg MA, Dutta S, Bawa S, Singh NK, Dutta SH. Recent scenario on psychotropic drug usage pattern among patients attending psychiatric outpatient department of a tertiary care teaching hospital in Nepal. IJBCP Int J Basic Clin Pharmacol. 2016;5(6):2649-52.

3. Kumar PP, Konwar M, Das S. To study the prescribing pattern of antipsychotic drugs in a tertiary care hospital of Assam. International Journal of Pharmacy and Pharmaceutical sciences. 2014;6(4):435-7.

4. Thakkar KB, Jain MM, Billa G, Joshi A, Khobragade AA. A drug utilization study of psychotropic drugs prescribed in the psychiatry outpatient department of a tertiary care hospital. J Clin Diagnostic Res. 2013;7(12):2759-64.

5. Goyal DV, Munjal S, Gupta DR. Drug Utilization Pattern of Psychotropic Drugs Prescribed in the Psychiatric Department of a Tertiary Care Government Hospital, Rajasthan. IOSR J Dent Med Sci. 2016;15(7):80-7.

6. Dutta S, Kaul V, Beg MA, Sindhu S, Singh NK, Dutta $\mathrm{S}$, et al. A psychotropic drug use study among depression patients attending private psychiatric practitioners of Dehradun, Uttarakhand. International Journal of Medical Science and Public Health. 2015;4(5):634-8

7. Chuki P, Khanapure A, De Sousa A. A study on prescribing patterns of atypical antipsychotic in psychiatric disorders International Journal of Pharma Research and Health Sciences. 2014;2(4):302-6.

8. Kurmi P, Paul PK, Dutta SK, Das S. To Study the Pattern of Adverse Drug Reaction of Antipsychotic Drugs in a Tertiary Care Hospital of Assam. International Journal of Pharmatech Research. 2015;8(1):101-5.

9. Sengupta G, Bhowmick S, Hazra A, Datta A, Rahaman M. Adverse drug reaction monitoring in psychiatry out-patient department of an Indian teaching hospital. IJP. 2011;43(1):36-9.

10. Swamy MK, Wagle L, Giri V. Drug utilisation pattern of psychotropic drugs in psychiatric outpatient department of rural tertiary care teaching hospital. International Journal of Pharmaceutical Sciences and Research. 2016;7(8):3497-508.

Cite this article as: Perumal VM, Bouddh SK, Nirmal SR, Deshpande A, Singh J, Prabhu N. Drug utilization study and prescribing patterns in psychiatry patients at a tertiary care hospital. Int $\mathbf{J}$ Basic Clin Pharmacol 2018;7:774-7. 